\title{
Stereotactic diffusion tensor imaging tractography for Gamma Knife radiosurgery
}

\author{
Cormac G. Gavin, FRCS(I), FRCS(SN), and H. lan Sabin, BMSc, FRCS(Eng), FRCS(Ed) \\ Gamma Knife Centre, St. Bartholomew's Hospital, London, United Kingdom
}

OBJECTIVE The integration of modern neuroimaging into treatment planning has increased the therapeutic potential and safety of stereotactic radiosurgery. The authors report their method of integrating stereotactic diffusion tensor imaging (DTI) tractography into conventional treatment planning for Gamma Knife radiosurgery (GKRS). The aim of this study was to demonstrate the feasibility of this technique and to address some of the technical limitations of previously reported techniques.

METHODS Twenty patients who underwent GKRS composed the study cohort. They consisted of 1 initial test case (a patient with a vestibular schwannoma), 5 patients with arteriovenous malformations, 9 patients with cerebral metastases, 1 patient with parasagittal meningioma, and 4 patients with vestibular schwannoma. DT images were obtained at the time of standard GKRS protocol MRI (T1 and T2 weighted) for treatment, with the patient's head secured by a Leksell stereotactic frame. All studies were performed using a 1.5-T magnet with a single-channel head coil. DTI was performed with diffusion gradients in 32 directions and coregistered with the volumetric T1-weighted study. DTI postprocessing by means of commercially available software allowed tensor computation and the creation of directionally encoded color-, apparent diffusion coefficient-, and fractional anisotropy-mapped sequences. In addition, the software allowed visualized critical tracts to be exported as a structural volume and integrated into GammaPlan as an "organ at risk" during shot planning. Combined images were transferred to GammaPlan and integrated into treatment planning.

RESULTS Stereotactic DT images were successfully acquired in all patients, with generation of correct directionally encoded color images. Tract generation with the software was straightforward and reproducible, particularly for axial tracts such as the optic radiation and the arcuate fasciculus. Corticospinal tract visualization was hampered by some artifacts from the base of the stereotactic frame, but this was overcome by a combination of frame/MRI volume adjustment and DTI seeding parameters. Coregistration of the DTI series with the T1-weighted treatment volume at the time of imaging was essential for the generation of correct tensor data. All patients with the exception of the vestibular schwannoma cases had treatment pathology in the vicinity of eloquent tracts and/or the cortex. No new neurological deficits due to radiation were recorded at the short-term follow-up.

CONCLUSIONS Recent reports in the medical literature have suggested that white matter tracts (particularly the optic radiation and arcuate fasciculus) are more vulnerable to radiation during stereotactic radiosurgery than previously thought. Integration of stereotactic tractography into GKRS represents a promising tool for preventing GKRS complications by reduction in radiation doses to functional organs at risk, including critical cortical areas and subcortical white matter tracts.

http://thejns.org/doi/abs/10.3171/2016.8.GKS161032

KEY WORDS Gamma Knife; stereotactic radiosurgery; diffusion tensor imaging; tractography; treatment planning; arteriovenous malformations

$\mathrm{G}$ AMMA Knife radiosurgery (GKRS), since its inception by Leksell, has been dependent on highquality imaging for its success. Developments in neuroimaging have paralleled the evolution in techniques and indications for stereotactic radiosurgery.

The integration of modern neuroimaging into treatment planning has increased the therapeutic potential and safety of stereotactic radiosurgery. Nevertheless, radiation-induced neuropathy remains a serious concern, particularly with arteriovenous malformations (AVMs) in eloquent locations. ${ }^{1,8}$

Previous reports have focused on using separately ac-

ABBREVIATIONS ADC = apparent diffusion coefficient; $\mathrm{AVM}=$ arteriovenous malformation; $\mathrm{CST}=$ corticospinal tract; $\mathrm{DEC}=$ directionally encoded color; $\mathrm{DTI}=$ diffusion tensor imaging; FA = fractional anisotropy; GKRS = Gamma Knife radiosurgery; MPR = multiplanar reformatting; OR = optic radiation; R/VOI = region/volume of interest. SUBMITTED June 12, 2016. ACCEPTED August 9, 2016. 
TABLE 1. Clinical characteristics of 20 patients who underwent stereotactic DTI tractography for GKRS

\begin{tabular}{|c|c|c|c|c|}
\hline Case No. & Age (yrs) & Pathology & Location & Clinical Presentation \\
\hline 1 & 47 & Vestibular schwannoma & Rt CPA & Hearing loss \\
\hline 2 & 72 & Metastasis $(n=1)$ from SCLC & Rt precentral & Incidental on surveillance MRI \\
\hline 3 & 47 & AVM & Lt occipital & Tinnitus \\
\hline 4 & 50 & AVM & Rt temporal & Hemorrhage, It superior quadrantanopia \\
\hline 5 & 58 & Vestibular schwannoma & CPA & Tinnitus \\
\hline 6 & 82 & Vestibular schwannoma & CPA & Tinnitus \\
\hline 7 & 55 & Metastases $(n=4)$ from renal cell carcinoma & Lt parietal & Incidental on surveillance MRI \\
\hline 8 & 72 & Vestibular schwannoma & Rt CPA & Hearing loss \\
\hline 9 & 24 & AVM & Occipital & Headache \\
\hline 10 & 29 & Metastasis $(n=1)$ from melanoma & Basal ganglia & Incidental on surveillance MRI \\
\hline 11 & 32 & AVM & Rt occipital & Tinnitus \\
\hline 12 & 74 & Vestibular schwannoma & CPA & Deafness \& ataxia \\
\hline 13 & 65 & Metastasis (solitary) & Intraventricular & Incidental on surveillance MRI \\
\hline 14 & 67 & Meningioma (Grade I) & Parasagittal & Seizure \& It sensorimotor deficit \\
\hline 15 & 43 & Metastases $(n=7)$ from melanoma & Lt parietal \& multiple other locations & Rt sensorimotor deficit \\
\hline 16 & 48 & Metastases $(n=2)$ from melanoma & Lt frontal \& cerebellar & Mild rt sensorimotor deficit \\
\hline 17 & 59 & Metastases $(n=7)$ from SCLC & Brainstem \& multiple other locations & Incidental on surveillance MRI \\
\hline 18 & 38 & AVM & Lt frontotemporal & Tinnitus \\
\hline 19 & 59 & Metastases $(n=2)$ from melanoma & Lt posterior frontal \& rt frontal & Incidental on surveillance MRI \\
\hline 20 & 56 & Metastases $(n=2)$ from NSCLC & Rt occipital \& It thalamic & Hemianopia \& mild rt upper-limb weakness \\
\hline
\end{tabular}

$\mathrm{CPA}=$ cerebellopontine angle; NSCLC = non-small cell lung cancer; $\mathrm{SCLC}=$ small-cell lung cancer.

quired tractography images and fusing them with stereotactic treatment images in order to analyze dosimetry to critical fiber tracts and improve treatment planning. ${ }^{11,12,13}$ This process has also been recently reported using other radiosurgical platforms..$^{5,16}$ The use of diffusion tensor imaging (DTI) has been reported to reduce the risk of motor complications after radiosurgery. ${ }^{9}$

We present our method of integrating stereotactic DTI tractography into conventional treatment planning for GKRS.

\section{Methods}

Twenty patients who underwent GKRS made up the study cohort. They consisted of 1 initial test case (a patient with a vestibular schwannoma), 5 patients with AVMs, 9 patients with cerebral metastases, 1 patient with parasagittal meningioma, and 4 patients with vestibular schwannoma. The clinical characteristics of the patients are listed in Table 1.

\section{Stereotactic 3D Imaging}

On the day of GKRS, the patient was immobilized in a Leksell model G, MRI-compatible stereotactic frame for stereotactic 3D anatomical MRI. All studies were performed using a 1.5-T magnet (Achieva; Philips) with a single-channel transmit/receive head coil. Our standard GKRS treatment protocol, consisting of T1- and T2weighted sequences (with contrast), was performed. The T1-weighted 3D fast field echo MRI study consisted of 100 sequential, 1.5-mm-thick axial slices with a resolution of $256 \times 256$ pixels over an FOV of $210 \mathrm{~mm}$ with 3D T2- weighted $512 \times 512-$ matrix 1.5-mm-thick axial slices as required. In the cases of AVMs, cerebral stereotactic angiography was also performed. The T1- and T2-weighted treatment sequences were transferred to GammaPlan via a picture-archiving and communication system for treatment planning.

\section{Stereotactic DTI}

High-directional DTI was performed at the time of standard GKRS protocol MRI (T1 and T2 weighted) for treatment, with the patient's head secured by a Leksell stereotactic frame and our standard "birdcage" transmit/ receive head coil.

We used a high-directional resolution DTI sequence single-shot spin echo-echo planar sequence (TR 2700 msec, TE $160 \mathrm{msec}$ ), acquiring 60-68 interleaved, contiguous 2-mm-thick axial images with no cardiac triggering.

A data matrix of $512 \times 345$ pixels over an FOV of 210 $\times 210 \mathrm{~mm}$ was obtained. Diffusion gradients were applied in 32 noncollinear independent axes by using a b value of 0 and $1000 \mathrm{sec} / \mathrm{mm}^{2}$. Each complete DTI data set required an additional 14-16 minutes of MR scanning time. The DTI and the T1- and T2-weighted data sets were transferred via CD to a StealthViz (Medtronic, Inc.) workstation for postprocessing.

\section{DTI Tractography}

DTI postprocessing was performed by means of commercially available software (StealthViz). This software is an additional component of established intraoperative neuronavigational software already in clinical use for pre- 


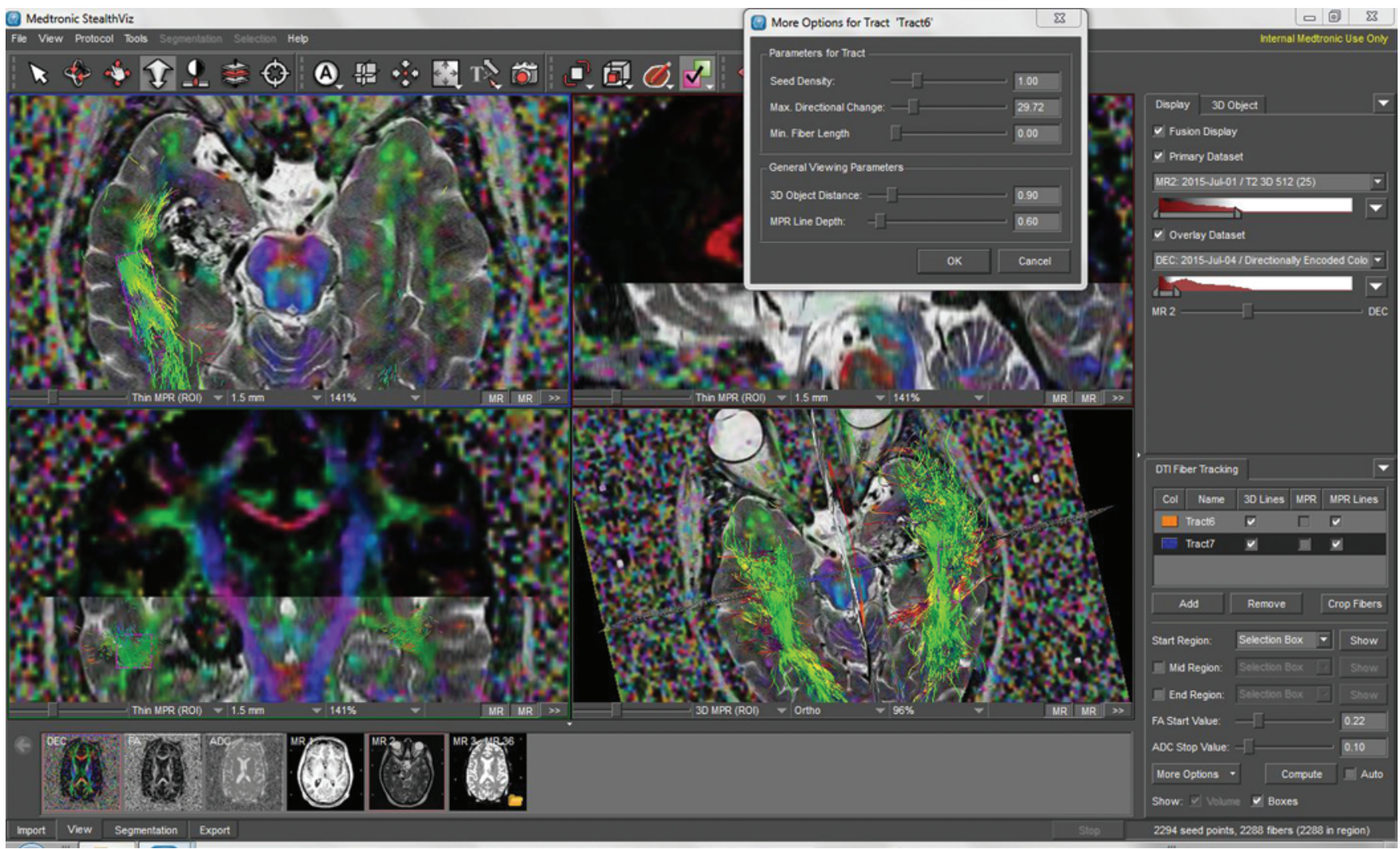

FIG. 1. Screenshot of the tractography graphic user interface; demonstration of bilateral OR tracts in a patient with a right-sided mesial temporal AVM with previous hemorrhage and established superior quadrantanopia.

operative planning and tract generation for cranial neurosurgery. ${ }^{4}$ It uses the deterministic Fiber Assignment by Continuous Tracking algorithm developed by Mori et al. at Johns Hopkins. ${ }^{14}$ This is a deterministic approach that uses the average axonal orientation within a voxel to estimate axonal projections, based on user-defined variables such as the fractional anisotropy (FA) and the maximum tract angle. Probabilistic algorithms, although allowing greater tract exploration into the gray matter, ${ }^{2}$ take much longer to compute and thus are not as amenable to interactive exploration of fiber tracts.

Several processing steps were required to build a DTI color map. These included gradient assignment, gradient registration, coregistration with the volumetric T1- and T2-weighted treatment sequences, and tensor computation. This resulted in the creation of FA-, apparent diffusion coefficient (ADC)-, and directionally encoded color (DEC)-mapped sequences. The image fusion function permitted visualization of the T1- or T2-weighted sequences with superimposition of the DEC sequences to aid fiber tracking (Fig. 1).

Tensor preparation is perhaps the most critical step in ensuring that the software generates anatomically correct tracts. Background masking is performed to eliminate air (represented by blue) by adjusting the thresholding. The correct gradient protocol must be selected according to the MR equipment manufacturer's protocol, e.g., Philips 32 gradient overplus off. The correct b value (1000) must also be selected per the protocol used. The gradient list must be checked to ensure correct alignment, and this is color coded. Incorrect gradient alignment will result in incorrect vector directions and erroneous tensor computation.

Gradient registration needs to ensure correct identification of the B0 sequence from the imported DTI series. Automatic coregistration of the DTI series with the B0 sequence is performed and verified. Coregistration involves selecting the T1-weighted treatment volume as the Reference Exam, and the B0 and T2-weighted sequences are then autoregistered and verified. Finally, tensor computation is performed, resulting in generation of the selected FA, DEC, and ADC sequences. It is important to verify correct colorization of the DEC sequence. The DEC sequence is viewed with either the T1- or T2-weighted sequence in fusion mode to aid identification of tract seeding points and to ensure the anatomical accuracy of the coregistration process.

DTI fiber tracking is performed by selecting the Fiber Tracking Tool, which permits the creation and display of tracts. Tract seeding was performed by defining a rectangular region/volume of interest (R/VOI) in the displayed coregistered data set. The definition of the R/VOI depended on the fiber structures to be displayed (i.e., optic radiation [OR], corticospinal tract [CST], and so on).

The methods for R/VOI selection of the major white fiber tracts are well described in the literature. ${ }^{3,15}$ The default value of angulation threshold was $45^{\circ}$, and the FA threshold was 0.20 . Midpoints and end points can also be chosen, depending on the tract to be visualized. 


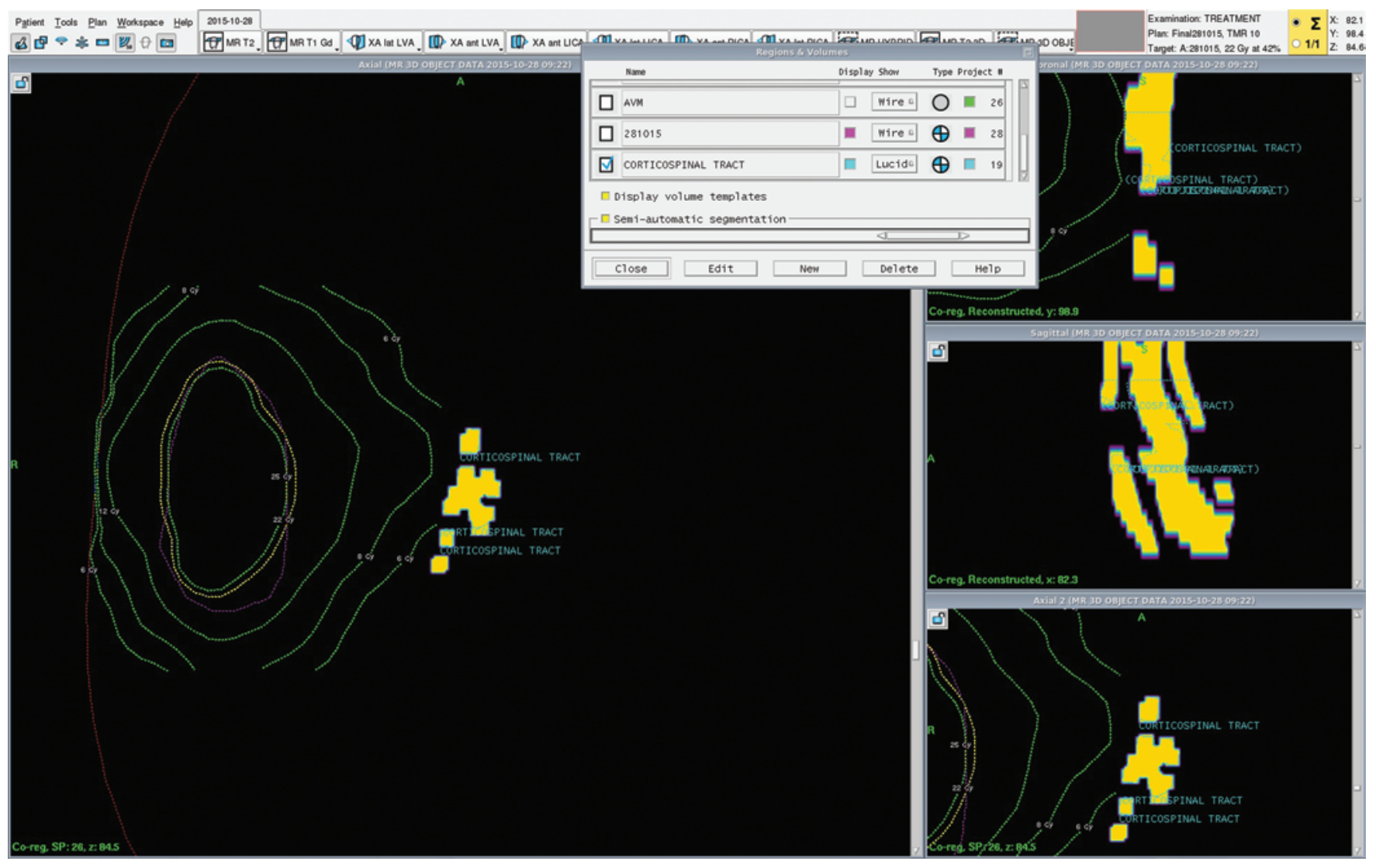

FIG. 2. Screenshot of an imported CST with segmentation in GammaPlan and creation as a volume at risk.

To complete the process, the generated tracts were rendered as 3D objects by selecting multiplanar reformatting (MPR) in the Fiber Tracking Tool and by clicking the Surf Box in the 3D Object Tool Card to view the tract. The 3D Tract Object could be edited with the Segmentation Screen.

The generated tracts were exported with the navigation examination (T1-weighted) and a hybrid examination (T1weighted with superimposed/burned 3D tract) in DICOM format using the 3D Object Data dialog. Tract studies were burned to DICOM CD for transfer to GammaPlan.

\section{Integration Into GammaPlan}

With GammaPlan open and with the standard treatment T1- and T2-weighted images already imported and verified, the $\mathrm{CD}$ containing the tract data was uploaded to the treatment workstation. The tractography data set was imported into GammaPlan (version 10.1; Elekta Instruments $\mathrm{AB}$ ) using the native DICOM CD import function. The DICOM data sets were imported into the treatment planning session and coregistered with the main T1weighted stereotactic treatment volume.

The imported tract images appeared in gray scale in the DICOM format and were color coded with GammaPlan to facilitate clear identification. The GammaPlan software allowed critical tracts to be segmented as a structural volume and integrated into GammaPlan as an "organ at risk" during shot planning (Fig. 2). Furthermore, tract volumes could be subjected to standard dosimetric analysis using the measure function. A summary of the steps required to fully integrate DTI data into GammaPlan is given in Fig. 3.

\section{Results}

Stereotactic DTI tractography was successful in generating the appropriate ADC, FA, and DEC sequences in all except for the first test case (19 of 20 cases). It was recognized that the first test case error was due to a rotational mismatch between the orientation of the DTI volume and the stereotactic T1-weighted treatment volume at the time of image acquisition. This error was overcome by creating an MRI protocol, which matched the spatial orientation of the DTI volume with the 3D stereotactic T1-weighted volume at scanning.

All successful cases were reviewed on the tractography workstation at the time of GKRS treatment planning to assess any pertinent anatomy or structures at risk. The cases of vestibular schwannoma were used as controls for the major tracts and to develop proficiency with tract generation because it is recognized that the technique is operator dependent. Although tractography has previously been used to identify the facial nerve, it was not performed in this study.

In the case of cerebral metastases, we observed that tractography provided additional useful clinical information for treatment planning (Fig. 4), which accounted for a recent deterioration in the patient's clinical symptoms 


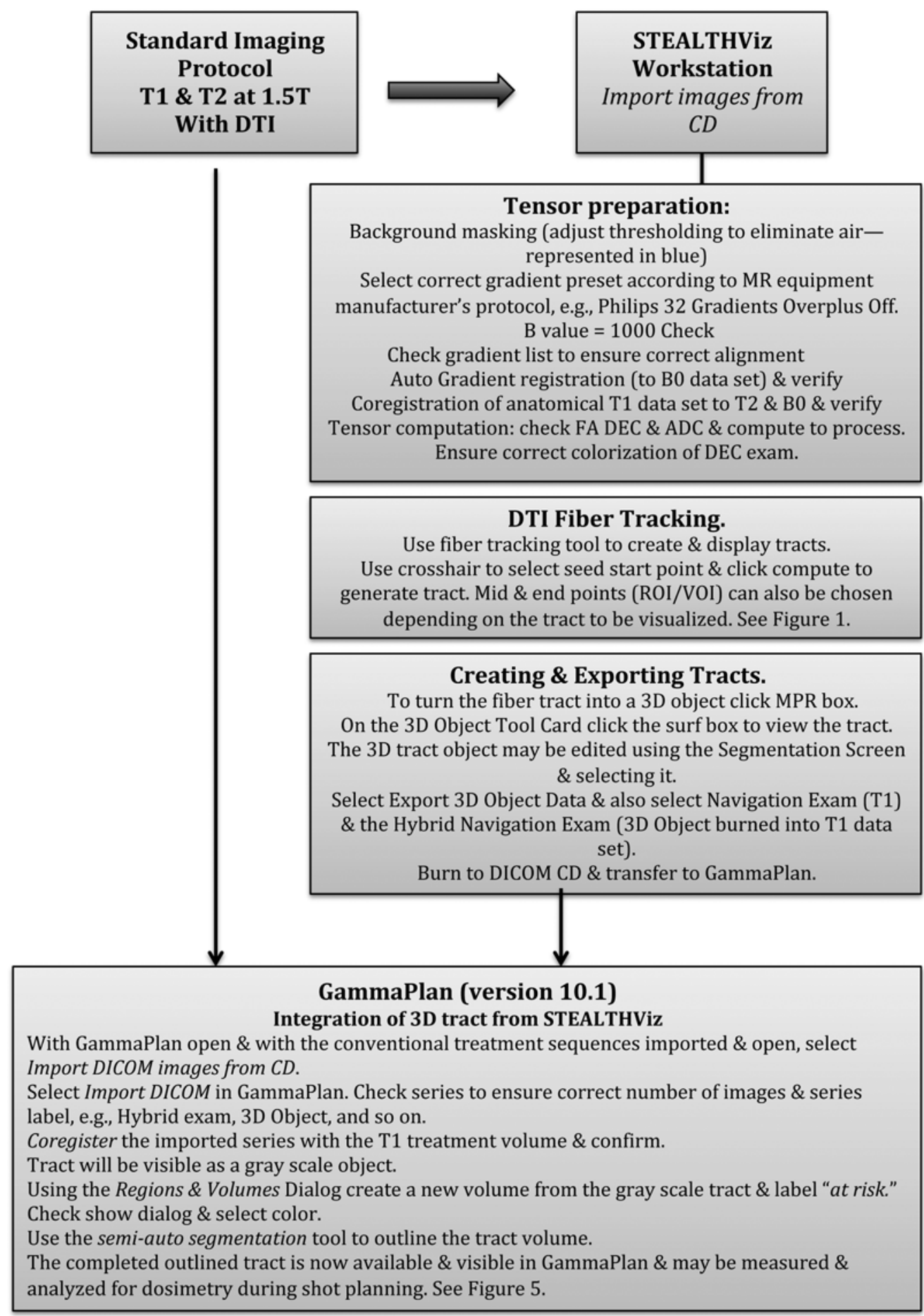

FIG. 3. Diagram summarizing the steps required to integrate stereotactic DTI into GammaPlan.

(hemianopia and hemiparesis). Tractography clearly demonstrated compression of the OR by the metastatic deposit and associated edema. Displacement and compression of the CST by a metastatic deposit and associated edema were also evident.

A selected solitary melanoma metastasis in proximity to the thalamus ( 25 Gy at $45 \%$ isodose) was selected for dosimetry with $\leq 1 \%$ of the CST volume receiving > 12 Gy with a maximum dose of $25 \mathrm{~Gy} \cdot{ }^{10}$ The patient did not develop any neurological deficits and was treated for a new contralateral hemispheric deposit 7 months later with demonstrated good radiological response to her previous treatment.

All 5 cases of AVMs had their selected generated tracts 

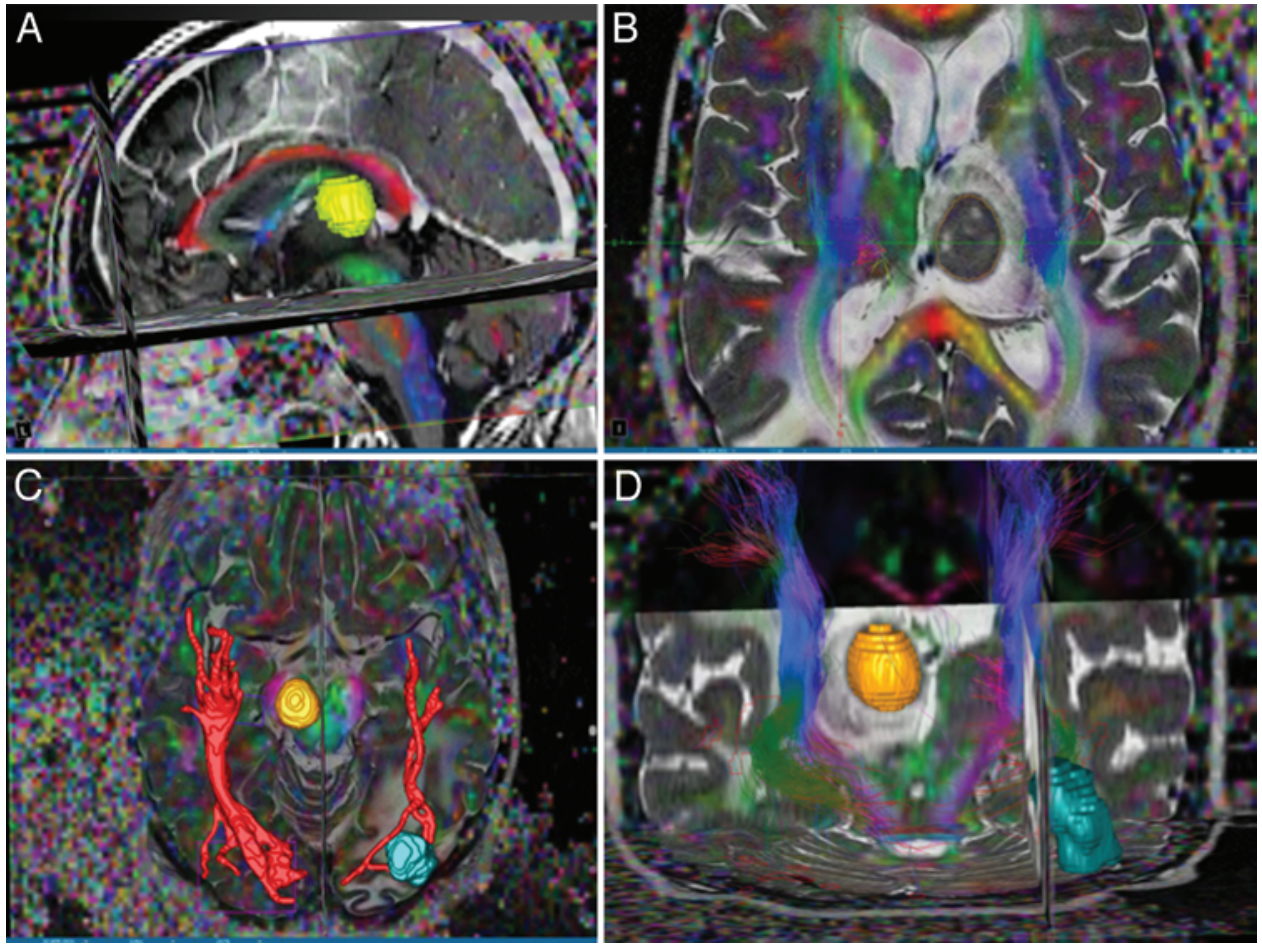

FIG. 4. OR compression and CST displacement demonstrated by DTI. The patient presented with progressive hemianopia rightsided pronator drift with mild right-sided hemiparesis. The patient had metastatic lung cancer, with previous whole-brain radiation therapy for left thalamic deposit (resistant) (A and B). The patient underwent salvage GKRS with new right occipital deposit (C). The deposits received $19-20$ Gy to the $42 \%-48 \%$ isodose, achieving $98 \%-99 \%$ coverage of the lesions (D).

retrospectively imported into GammaPlan for dosimetric analysis of the tracts at risk. These data are summarized in Table 2. Our reported data are within the previously published tract tolerances summarized in Table 3.

One patient had staged (by feeding vessel) treatment of a large-volume AVM adjacent to the CST (Fig. 5). Followup at the second-stage treatment 6 months later demonstrated early nidus volume reduction and no neurological deficit.

Three of the AVMs are postgeniculate and pose risk of causing damage to the OR. ${ }^{3}$ During this very short followup period (6-12 months), none of the patients developed a new deficit as a result of their GKRS.

\section{Discussion}

Recent reports in the medical literature have suggested that white matter tracts (particularly the $\mathrm{OR}^{12}$ and arcuate fasciculus ${ }^{13}$ ) are more vulnerable to radiation during SRS than previously thought. Traditional estimates for the risk of radiation necrosis ${ }^{7}$ may not be predictive enough, especially when the pathology is adjacent to major fiber tracts. Integration of stereotactic tractography into GKRS represents a promising tool for preventing GKRS complications by reduction in radiation doses to functional organs at risk, including critical cortical areas and subcortical white matter tracts. This has been demonstrated by Koga et al., ${ }^{9}$ who reported that the integration of CST DTI into GKRS planning for AVM reduces the incidence of motor complications without compromising obliteration rates.

The use of DTI is not exclusive to GKRS, and other centers have reported integrating both DTI and functional MRI into linear accelerator systems to aid treatment planning. ${ }^{5,16}$ They have also reported problems with coregistration accuracy. ${ }^{5}$

TABLE 2. Treatment data of DTI with dosimetry for 5 patients with AVMs

\begin{tabular}{|c|c|c|c|c|c|}
\hline Case No. & Location & Prescription Dose \& Vol & Tract at Risk & Tract Dosimetry & Clinical Outcome \\
\hline 3 & Lt occipital & 20 Gy @ 43\%, 95\% of $7.21-\mathrm{cm}^{3}$ AVM & OR & Max dose 6.45 Gy & No clinical change \\
\hline $4^{*}$ & Rt temporal & 25 Gy @ 51\%, 96\% of 3.54-cm³ AVM & OR & $\begin{array}{l}\text { Max dose } 16.6 \text { Gy to Meyers loop; } \\
4 \text { Gy to optic nerve \& chiasm }\end{array}$ & No clinical change \\
\hline 9 & Rt occipital & 25 Gy @ 43\%, 95\% of $4.30-\mathrm{cm}^{3}$ AVM & OR & Max dose $11.40 \mathrm{~Gy}$ & No clinical change \\
\hline 11 & Rt occipital & 20 Gy @ 44\%, 95\% of $5.19-\mathrm{cm}^{3}$ AVM & OR & Max dose $5.5 \mathrm{~Gy}$ & No clinical change \\
\hline 18 & Rt frontotemporal & 22 Gy @ 42\%, 95\% of $14.36-\mathrm{cm}^{3}$ AVM & CST & Max dose $8.1 \mathrm{~Gy}$ & No clinical change \\
\hline
\end{tabular}

\footnotetext{
${ }^{*}$ Case 4 presented with hemorrhage and left superior quadrantanopia.
} 
TABLE 3. Review of DTI and tract tolerance in the GKRS literature

\begin{tabular}{ccc}
\hline Authors \& Year & Tract Studied & Tract Tolerance \\
\hline $\begin{array}{c}\text { Maruyama et } \\
\text { al., 2009 }\end{array}$ & Arcuate fasciculus & Frontal, $10 \mathrm{~Gy}$; temporal, 6 Gy \\
\hline $\begin{array}{c}\text { Maruyama et } \\
\text { al., 2007 }\end{array}$ & OR & $<8 \mathrm{~Gy}$ \\
\hline $\begin{array}{c}\text { Maruyama et } \\
\text { al., 2005 }\end{array}$ & CST & $\begin{array}{c}\text { Tract vol }<25 \mathrm{~Gy} \text {, maximum } \\
\text { dose 28 Gy }\end{array}$ \\
\hline $\begin{array}{c}\text { Maruyama et } \\
\text { al., 2008 }\end{array}$ & Pyramidal tract & $\begin{array}{c}\text { Pyramidal tract }<23 \text { Gy ideally } \\
\text { outside 20-Gy isodose; inter- } \\
\text { nal capsule more sensitive }\end{array}$ \\
\hline
\end{tabular}

Our reported technique of stereotactic DTI goes some way toward improvement upon the limitations of previously reported techniques. The inherent problem of spatial accuracy and distortion induced by the coregistration process when DT images are acquired nonstereotactically is improved upon by acquiring the images stereotactically at the time of treatment imaging. Ensuring that the spatial orientation of the DTI volume is matched with the 3D stereotactic T1-weighted volume at MRI is a critical step for correct gradient alignment.

Correct gradient alignment is required for correct vector direction and is the key to tensor computation. It also ensures proper coregistration with the T1-weighted treat- ment series. The postprocessing takes approximately an additional 15 minutes plus another 10 minutes to integrate the tract data into GammaPlan (depending on the complexity of the tract shape). Another potential use of integrated DTI is that the Dynamic Shaping function of GammaPlan may be used to move treatment beams away from critical tracts when dosimetry demonstrates that the tolerance dose may be exceeded.

\section{Conclusions}

The utility of DTI in GKRS will no doubt benefit from advances in stereotactic frame design, which will allow the use of conventional multichannel coils and imaging at higher field strength with less image distortion. Further development is required to improve the integration of DTI into the treatment planning software and to increase the ease of operator use. DTI has limitations and is user dependent; it is likely to be superseded in the future by even higher-resolution techniques in tractography involving probabilistic techniques. ${ }^{6}$

The aim of our study was to demonstrate the feasibility of our technique and to address some of the technical limitations of previously reported techniques. Further studies using DTI are required to increase our knowledge of specific tract and critical cerebral structure radiation tolerances to better improve the therapeutic potential and safety of GKRS.

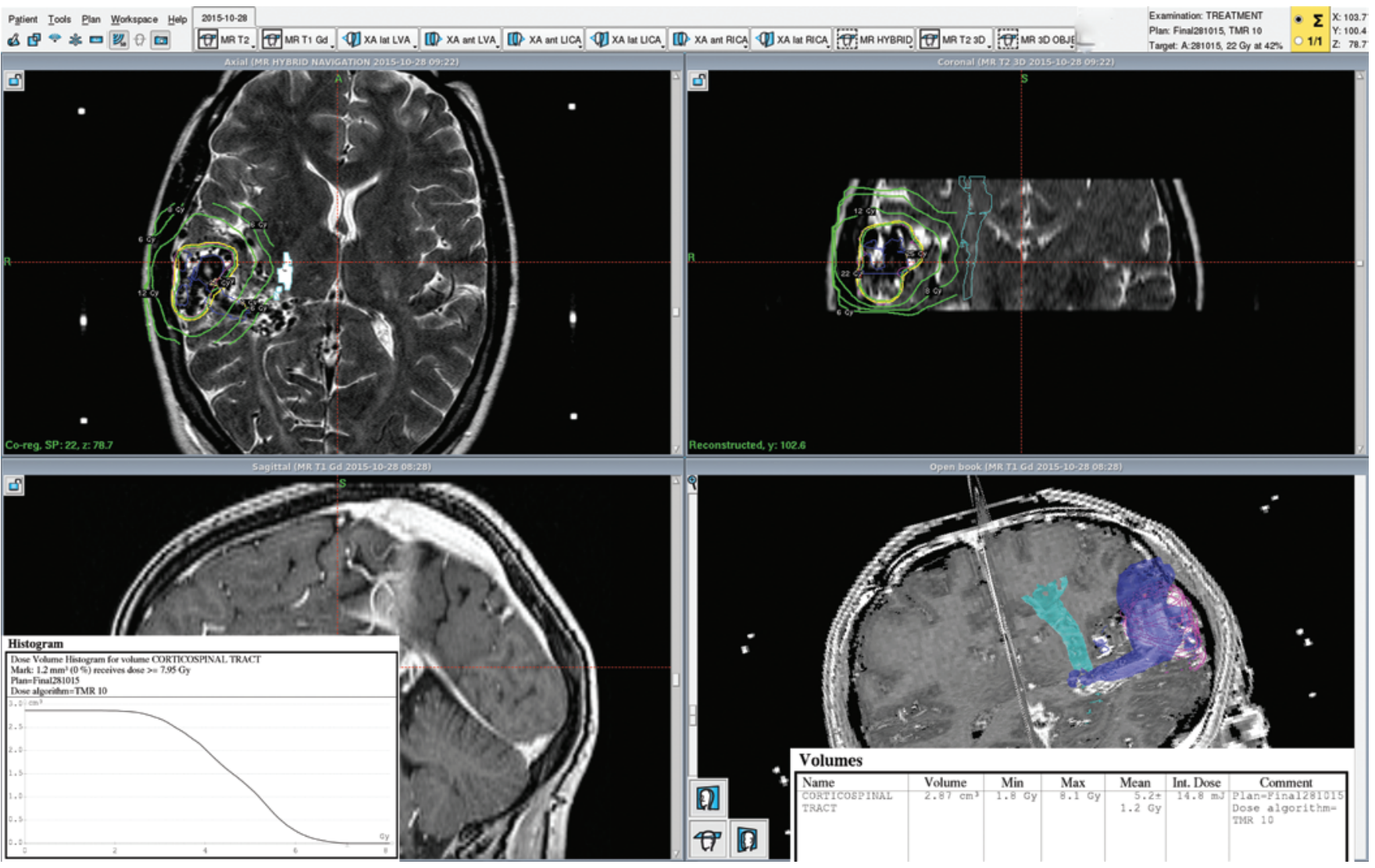

FIG. 5. Integration of DTI into treatment planning for AVM: 20 Gy at $42 \%$ isodose adjacent to the CST (maximum dose $8.1 \mathrm{~Gy}$ ). 


\section{Acknowledgments}

We are grateful to Marc Miquel, PhD, Lead MR Physicist at St. Bartholomew's Hospital, and the MRI radiographer team at St. Bartholomew's Hospital, without whom this study would not have been possible.

\section{References}

1. Andrade-Souza YM, Zadeh G, Scora D, Tsao MN, Schwartz ML: Radiosurgery for basal ganglia, internal capsule, and thalamus arteriovenous malformation: clinical outcome. Neurosurgery 56:56-64, 2005

2. Behrens TE, Woolrich MW, Jenkinson M, Johansen-Berg H, Nunes RG, Clare S, et al: Characterization and propagation of uncertainty in diffusion-weighted MR imaging. Magn Reson Med 50:1077-1088, 2003

3. Bowden G, Kano H, Caparosa E, Tonetti D, Niranjan A, Monaco EA III, et al: Stereotactic radiosurgery for arteriovenous malformations of the postgeniculate visual pathway. J Neurosurg 122:433-440, 2015

4. Cho JM, Kim EH, Kim J, Lee SK, Kim SH, Lee KS, et al: Clinical use of diffusion tensor image-merged functional neuronavigation for brain tumor surgeries: review of preoperative, intraoperative, and postoperative data for 123 cases. Yonsei Med J 55:1303-1309, 2014

5. Conti A, Pontoriero A, Ricciardi GK, Granata F, Vinci S, Angileri FF, et al: Integration of functional neuroimaging in CyberKnife radiosurgery: feasibility and dosimetric results. Neurosurgical Focus 34(4):E5, 2013

6. Farquharson S, Tournier JD, Calamante F, Fabinyi G, Schneider-Kolsky M, Jackson GD, et al: White matter fiber tractography: why we need to move beyond DTI. J Neurosurg 118:1367-1377, 2013

7. Flickinger JC, Kondziolka D, Lunsford LD, Kassam A, Phuong LK, Liscak R, et al: Development of a model to predict permanent symptomatic postradiosurgery injury for arteriovenous malformation patients. Int J Radiat Oncol Biol Phys 46:1143-1148, 2000

8. Flickinger JC, Kondziolka D, Lunsford LD, Pollock BE, Yamamoto M, Gorman DA, et al: A multi-institutional analysis of complication outcomes after arteriovenous malformation radiosurgery. Int J Radiat Oncol Biol Phys 44:67-74, 1999

9. Koga T, Shin M, Maruyama K, Kamada K, Ota T, Itoh D, et al: Integration of corticospinal tractography reduces motor complications after radiosurgery. Int J Radiat Oncol Biol Phys 83:129-133, 2012

10. Maruyama K, Kamada K, Ota T, Koga T, Itoh D, Ino K, et al: Tolerance of pyramidal tract to Gamma Knife radiosurgery based on diffusion-tensor tractography. Int J Radiat Oncol Biol Phys 70:1330-1335, 2008
11. Maruyama K, Kamada K, Shin M, Itoh D, Aoki S, Masutani $\mathrm{Y}$, et al: Integration of three-dimensional corticospinal tractography into treatment planning for Gamma Knife surgery. J Neurosurg 102:673-677, 2005

12. Maruyama K, Kamada K, Shin M, Itoh D, Masutani Y, Ino $\mathrm{K}$, et al: Optic radiation tractography integrated into simulated treatment planning for Gamma Knife surgery. J Neurosurg 107:721-726, 2007

13. Maruyama K, Koga T, Kamada K, Ota T, Itoh D, Ino K, et al: Arcuate fasciculus tractography integrated into Gamma Knife surgery. J Neurosurg 111:520-526, 2009

14. Mori S, Crain BJ, Chacko VP, van Zijl PC: Three-dimensional tracking of axonal projections in the brain by magnetic resonance imaging. Ann Neurol 45:265-269, 1999

15. Mori S, van Zijl PC: Fiber tracking: principles and strategies - a technical review. NMR Biomed 15:468-480, 2002

16. Pantelis E, Papadakis N, Verigos K, Stathochristopoulou I, Antypas C, Lekas L, et al: Integration of functional MRI and white matter tractography in stereotactic radiosurgery clinical practice. Int J Radiat Oncol Biol Phys 78:257-267, 2010

17. Thomas B, Eyssen M, Peeters R, Molenaers G, Van Hecke P, De Cock P, et al: Quantitative diffusion tensor imaging in cerebral palsy due to periventricular white matter injury. Brain 128:2562-2577, 2005

\section{Disclosures}

Mr. Sabin is a consultant for HCA International.

\section{Author Contributions}

Conception and design: Gavin. Acquisition of data: Gavin. Analysis and interpretation of data: Gavin. Drafting the article: Gavin. Critically revising the article: both authors. Reviewed submitted version of manuscript: Gavin. Approved the final version of the manuscript on behalf of both authors: Gavin. Study supervision: Sabin.

\section{Supplemental Information \\ Previous Presentations}

Portions of this work were presented as a plenary oral presentation (abstract no. 147) at the 18th International Leksell Gamma Knife Society meeting in Amsterdam, the Netherlands, May 15-19, 2016.

\section{Correspondence}

Cormac G. Gavin, The London Gamma Knife Centre at Bart's, St. Bartholomew's Hospital, West Smithfield, London EC1A 7BE, United Kingdom.email: c.gavin@mac.com. 